\title{
Classification of external Zonotopal algebras
}

\author{
Gleb Nenashev* \\ Department of Mathematics \\ Massachusetts Institute of Technology \\ Cambridge, U.S.A. \\ memashev@mit.edu
}

Submitted: Nov 7, 2018; Accepted: Dec 2, 2018; Published: Mar 8, 2019

(c) The author. Released under the CC BY-ND license (International 4.0).

\begin{abstract}
In this paper we work with power algebras associated to hyperplane arrangements. There are three main types of these algebras, namely, external, central, and internal zonotopal algebras. We classify all external algebras up to isomorphism in terms of zonotopes. Also, we prove that unimodular external zonotopal algebras are in one to one correspondence with regular matroids. For the case of central algebras we formulate a conjecture.
\end{abstract}

Mathematics Subject Classifications: 05B35; 05C05; 05E40; 13P99

\section{Introduction}

In this paper we work with power algebras, which are the quotients of polynomial rings by power ideals. In particular, we consider zonotopal ideals, which are associated to zonotopes. These ideals were independently introduced in two different ways. The first definition was used by F. Ardila and A. Postnikov [2]; it originates from the algebras generated by the curvature forms of tautological Hermitian linear bundles [4, 29], see also papers $[5,6,15,16,17,23,24,27,28,30]$, where the quotient algebras by these ideals were discussed by details. At the same time a similar definition and the term were established by O. Holtz and A. Ron [13]; Their definitions originates from Box-Splines and from Dahmen-Micchelli's space [1, 9, 11], see also the papers [10, 12, 14, 19, 20, 21, 22, 31].

More concretely, let $A \in \mathbb{R}^{n \times m}$ be a matrix of rank $n$. Denote by $y_{1}, \ldots, y_{m} \in \mathbb{R}^{n}$ the columns and by $t_{1}, \ldots, t_{n} \in \mathbb{R}^{m}$ the rows of $A$. For a matrix $A$, we define the zonotope

$$
Z_{A}:=\bigoplus_{i \in[m]}\left[0, y_{i}\right] \subset \mathbb{R}^{n}
$$

*Supported by the National Science Foundation under Grant DMS-1440140 while the author was staying at the Mathematical Sciences Research Institute in Berkeley, California. 
as the Minkovskii sum of intervals $\left[0, y_{i}\right], i \in[m]$. By $\mathcal{F}(A)$ we denote the set of facets of $Z_{A}$. For any facet $H \in \mathcal{F}(A)$, we define $m(H)$ as the number of non-zero coordinates of the vector $\eta_{H} A \in \mathbb{R}^{m}$, where $\eta_{H} \in \mathbb{R}^{n}$ is a normal vector to $H$.

Let $\mathcal{C}_{A}^{(k)}$ be the quotient algebra

$$
\mathcal{C}_{A}^{(k)}:=\mathbb{R}\left[x_{1}, \ldots, x_{n}\right] / \mathcal{I}_{A}^{(k)},
$$

where $\mathcal{I}_{A}^{(k)}$ is the zonotopal ideal generated by the polynomials

$$
p_{H}^{(k)}=\left(\eta_{h} \cdot\left(x_{1}, \ldots, x_{n}\right)\right)^{m(H)+k}, \quad H \in \mathcal{F}(A) .
$$

Here $\eta_{h} \cdot\left(x_{1}, \ldots, x_{n}\right)$ is the linear form in $x_{i}, i \in[n]$ obtained by taking the scalar product.

There are 3 main cases studied of the above construction, where $k= \pm 1$ and 0 ; they were considered in $[2,13]$.

- $k=1: \quad \mathcal{C}_{A}^{\mathcal{E} x}=\mathcal{C}_{A}^{(1)}$ is the external zonotopal algebra for $A$;

- $k=0: \quad \mathcal{C}_{A}^{C}=\mathcal{C}_{A}^{(0)}$ is the central zonotopal algebra for $A$;

- $k=-1: \quad \mathcal{C}_{A}^{\mathcal{I n}}=\mathcal{C}_{A}^{(-1)}$ is the internal zonotopal algebra for $A$.

Remark 1 . The case $k>1$ is not "zonotopal", because the ideal $\hat{\mathcal{I}}^{(k)}$ generated by

$$
p_{h}=\left(h \cdot\left(x_{1}, \ldots, x_{n}\right)\right)^{m(h)+k}, \quad h \in \mathbb{R}^{n}
$$

is different from $\mathcal{I}^{(k)}$. They coincide only in the case when $k \leqslant 1$.

In the case $k \leqslant-5$, the Hilbert series is not a specialization of the corresponding Tutte polynomial, see [2].

Theorem 2 (cf. [2, 5, 13, 21], external case [28], central case for graphs [27]). For a matrix $A \in \mathbb{R}^{n \times m}$, the Hilbert series of the zonotopal algebras are given by

- $\mathcal{H}\left(\mathcal{C}_{A}^{\mathcal{E} x}\right)=q^{m-n} T_{A}\left(1+q, \frac{1}{q}\right)$;

- $\mathcal{H}\left(\mathcal{C}_{A}^{C}\right)=q^{m-n} T_{A}\left(1, \frac{1}{q}\right)$;

- $\mathcal{H}\left(\mathcal{C}_{A}^{\mathcal{I} n}\right)=q^{m-n} T_{A}\left(0, \frac{1}{q}\right)$,

where $T_{A}$ is the Tutte polynomial of the vector configuration of the columns of $A$ (i.e., vectors $\left.y_{1}, \ldots, y_{m}\right)$.

There are other definitions of external algebras in [28]. Let $\Phi_{m}$ be the square-free commutative algebra generated by $\phi_{i}, i \in[m]$, i.e., by relations

$$
\phi_{i} \phi_{j}=\phi_{j} \phi_{i}, i, j \in[m] \text { and } \phi_{i}^{2}=0, i \in[m] .
$$


Theorem 3 (cf. [28]). The external algebra $\mathcal{C}_{A}^{\mathcal{E} x}$ is isomorphic to the subalgebra of $\Phi_{A}^{\mathcal{E} x}:=$ $\Phi_{m}$ generated by

$$
X_{i}:=t_{i} \cdot\left(\phi_{1}, \ldots, \phi_{m}\right), i \in[n] .
$$

According to the works [3] and [2] there is a similar definition of central zonotopal algebras. In papers $[16,17]$ we obtained the analog of the Theorem 3 in the case of internal zonotopal algebras for totally unimodular matrices, see the definition below.

The main interesting examples of zonotopal algebras arise for totally unimodular matrices and for graphs. Namely, a matrix $A$ is totally unimodular if any its minors is equal to \pm 1 or 0 . In this case, the total dimensions of the algebras have a nice interpretation.

Theorem 4 (cf. [13]). Let $A \in \mathbb{R}^{n \times m}$ be a totally unimodular matrix of rank $n$. Then the following holds:

- $\operatorname{dim}\left(\mathcal{C}_{A}^{\mathcal{E x}}\right)$ is equal to the number of lattice points of $Z_{A}$;

- $\operatorname{dim}\left(\mathcal{C}_{A}^{C}\right)$ is equal to the volume of $Z_{A}$;

- $\operatorname{dim}\left(\mathcal{C}_{A}^{\mathcal{I} n}\right)$ is equal to the number of interior lattice points of $Z_{A}$.

The main examples of totally unimodular matrices are graphs. Namely, let $G$ be a graph on $n$ vertices, then the incidence matrix of any orientation of $G$ is totally unimodular. To construct the zonotopal algebra, we should forget exactly one row for each connected component of $G$. These algebras are independent (up to isomorphism) of the choice of orientations and rows.

These graphical algebras were considered in [15, 16, 23, 24, 28]. In the graphical case Theorem 4 can be written in graph theoretical terminology.

Theorem 5 (cf. [27]). Let $G$ be a graph. Then the following holds:

- $\operatorname{dim}\left(\mathcal{C}_{G}^{\mathcal{E} x}\right)$ is equal to the number of forests in $G$;

- $\operatorname{dim}\left(\mathcal{C}_{G}^{C}\right)$ is equal to the number of trees in $G$ (in the connected case).

It is well-known that the number of lattice points (volume) of the corresponding zonotope and the number of forests (trees) of a graph are the same, see for example [7, 18]. (Points of the zonotope correspond to score vectors).

Example 6. Let $G$ be graph on the vertex set $\{0,1,2\}$ having 4 edges

$$
(0,1),(0,2),(1,2) \text {, and }(1,2) \text {, }
$$

see fig. 1, left. Let us orient all edges from the smaller to the larger vertex. Consider the incidence matrix after forgetting of 0 -th row

$$
A:=\left[\begin{array}{cccc}
-1 & 0 & 1 & 1 \\
0 & -1 & -1 & -1
\end{array}\right]
$$

see zonotope $Z_{A}$ on fig. 1 , right.

The zonotope $Z_{A}$ has 6 facets. We need the set of its normals (note that parallel facets have the same normal up to a factor). There are 3 normals 

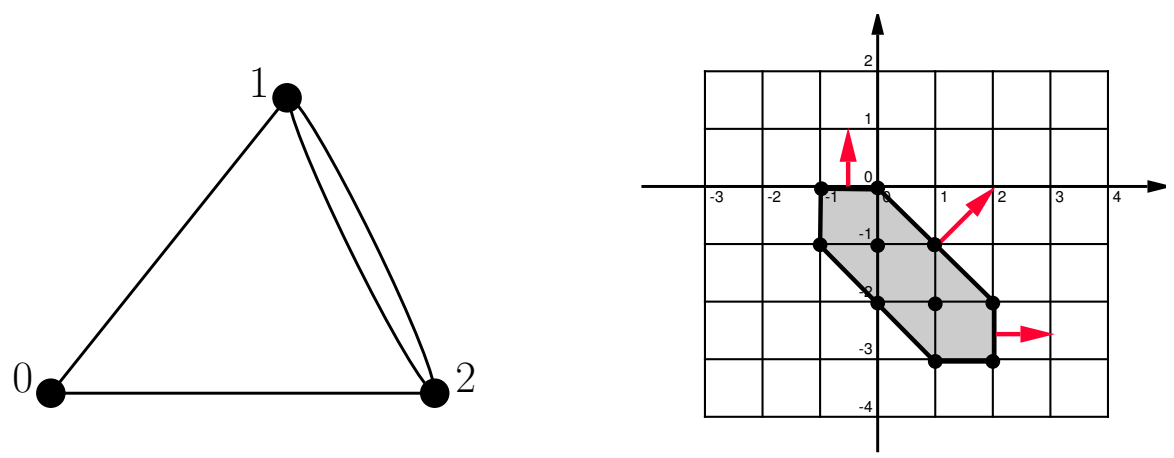

Figure 1: A graph and its corresponding zonotope.

- $\eta_{1}=(1,0)$;

- $\eta_{2}=(0,1)$;

- $\eta_{3}=(1,1)$.

It is easy to check that $m\left(\eta_{1}\right)=m\left(\eta_{2}\right)=3$ and $m\left(\eta_{3}\right)=2$. Hence,

$$
\mathcal{I}_{A}^{(k)}=\left\langle x_{1}^{3+k}, x_{2}^{3+k},\left(x_{1}+x_{2}\right)^{2+k}\right\rangle .
$$

Then

- $\mathcal{H}\left(\mathcal{C}_{A}^{\mathcal{E} x}\right)=1+2 q+3 q^{2}+3 q^{3}+q^{4} \quad$ and $\quad \operatorname{dim}\left(\mathcal{C}_{A}^{\mathcal{E} x}\right)=10$

- $\mathcal{H}\left(\mathcal{C}_{A}^{C}\right)=1+2 q+2 q^{2}$ and $\operatorname{dim}\left(\mathcal{C}_{A}^{C}\right)=5$;

- $\mathcal{H}\left(\mathcal{C}_{A}^{\mathcal{I} n}\right)=1+q$ and $\operatorname{dim}\left(\mathcal{C}_{A}^{\mathcal{I} n}\right)=2$.

It is easy to check that 10,5 , and 2 are exactly the number of lattice points, the area, and the number of interior lattice points of $Z_{A}$, respectively. Furthermore, 10 and 5 are the number of forests and trees in $G$. In this case the Tutte polynomial of the graph is given by $T_{G}(x, y)=T_{A}(x, y)=x+y+x^{2}+x y+y^{2}$.

The following important property of external graphical algebras has been proven in $[24]$.

Theorem 7 (cf. [24]). Given two graphs $G_{1}$ and $G_{2}$. Then the following are equivalent:

- $\mathcal{C}_{G_{1}}^{\mathcal{E} x}$ and $\mathcal{C}_{G_{2}}^{\mathcal{E} x}$ are isomorphic as non-graded algebras;

- $\mathcal{C}_{G_{1}}^{\mathcal{E x}}$ and $\mathcal{C}_{G_{2}}^{\mathcal{E} x}$ are isomorphic as graded algebras;

- the graphical matroids $M_{G_{1}}$ and $M_{G_{2}}$ are isomorphic.

Conjecture 8 (cf. [24]). Given two connected graphs $G_{1}$ and $G_{2}$, the following claims are equivalent: 
- $\mathcal{C}_{G_{1}}^{C}$ and $\mathcal{C}_{G_{2}}^{C}$ are isomorphic as non-graded algebras;

- $\mathcal{C}_{G_{1}}^{C}$ and $\mathcal{C}_{G_{2}}^{C}$ are isomorphic as graded algebras;

- the bridge-free matroids $M_{G_{1}}$ and $M_{G_{2}}$ are isomorphic.

Here the bridge-free matroid of a graph is its graphical matroid obtained after deleting all bridges.

In the paper [23], the $\mathcal{K}$-theoretic filtrations of external and central graphical algebras were considered, see definitions there. Denote by $\mathcal{K}_{G}^{\mathcal{E} x}$ the $\mathcal{K}$-theoretic filtration of $\mathcal{C}_{G}^{\mathcal{E} x}$.

Theorem 9 (cf. [23]). Given two graphs $G_{1}$ and $G_{2}$ without isolated vertices, the filtered algebras $\mathcal{K}_{G_{1}}^{\mathcal{E} x}$ and $\mathcal{K}_{G_{2}}^{\mathcal{E} x}$ are isomorphic if and only if $G_{1}$ and $G_{2}$ are isomorphic.

The structure of this paper is as follows: in $\S 2$ we present a classification of external zonotopal algebras and a conjecture for the central case; in $\S 3$ we prove our classification.

\section{Main results}

Definition 10. Two linear spaces $V_{1} \subset \mathbb{R}^{m_{1}}$ and $V_{2} \subset \mathbb{R}^{m_{2}}$ are called z-equivalent if $m_{1}=m_{2}=m$ and there is an invertible diagonal matrix $D \in \mathbb{R}^{m \times m}$ and a permutation $\pi \in S_{m}$ such that

$$
V_{1}=V_{2}(\pi D) \text {. }
$$

The matrices $A_{1} \in \mathbb{R}^{n_{1} \times m_{1}}$ of rank $n_{1}$ and $A_{2} \in \mathbb{R}^{n_{2} \times m_{2}}$ of rank $n_{2}$ are called $z$ equivalent if the span of rows of $A_{1}$ is $z$-equivalent to the span of rows of $A_{2}$.

Remark 11. It is easy to see that $z$-equivalence is an equivalence relation.

In the case when $A_{1}$ and $A_{2}$ do not have proportional columns, we can say that the matrix $A_{1}$ is equivalent to $A_{2}$ if and only if their zonotopes are equivalent (since we can reconstruct the "matrix" from the zonotope in this case).

This equivalence is weaker than that of matroids.

Proposition 12. If two matrices $A_{1}$ and $A_{2}$ are $z$-equivalent, then the matroids $M_{A_{1}}$ and $M_{A_{2}}$ are isomorphic.

It is easy to check that $\mathcal{C}_{A_{1}}^{\mathcal{E} x}$ and $\mathcal{C}_{A_{2}}^{\mathcal{E} x}$ are isomorphic if $A_{1}$ and $A_{2}$ are $z$-equivalent. The converse also holds.

Theorem 13. Let $A_{1} \in \mathbb{R}^{n_{1} \times m_{1}}$ and $A_{2} \in \mathbb{R}^{n_{2} \times m_{2}}$ be two matrices of rank $n_{1}$ and $n_{2}$ respectively. Then the following are equivalent:

- $\mathcal{C}_{A_{1}}^{\mathcal{E} x}$ and $\mathcal{C}_{A_{2}}^{\mathcal{E} x}$ are isomorphic as non-graded algebras;

- $\mathcal{C}_{A_{1}}^{\mathcal{E} x}$ and $\mathcal{C}_{A_{2}}^{\mathcal{E x}}$ are isomorphic as graded algebras; 
- $A_{1}$ and $A_{2}$ are z-equivalent.

Corollary 14. Let $A_{1} \in \mathbb{R}^{n_{1} \times m_{1}}$ and $A_{2} \in \mathbb{R}^{n_{2} \times m_{2}}$ be two matrices of ranks $n_{1}$ and $n_{2}$ respectively, with isomorphic external algebras $\mathcal{C}_{A_{1}}^{\mathcal{E} x} \cong \mathcal{C}_{A_{2}}^{\mathcal{E} x}$. Then the matroids $M_{A_{1}}$ and $M_{A_{2}}$ are isomorphic.

The following theorems shows that unimodular external zonotopal algebras are in one-to-one correspondence with regular matroids.

Theorem 15. Let $A_{1} \in \mathbb{R}^{n_{1} \times m_{1}}$ and $A_{2} \in \mathbb{R}^{n_{2} \times m_{2}}$ be two unimodular matrices of rank $n_{1}$ and $n_{2}$ respectively. Then the following are equivalent:

- $\mathcal{C}_{A_{1}}^{\mathcal{E x}}$ and $\mathcal{C}_{A_{2}}^{\mathcal{E} x}$ are isomorphic as non-graded algebras;

- $\mathcal{C}_{A_{1}}^{\mathcal{E} x}$ and $\mathcal{C}_{A_{2}}^{\mathcal{E x}}$ are isomorphic as graded algebras;

- $A_{1}$ and $A_{2}$ are z-equivalent.

- the matroids $M_{A_{1}}$ and $M_{A_{2}}$ are isomorphic.

Since for graphs we have a totally unimodular matrix, all graphical matroids are regular; the converse is almost true. Every regular matroid may be constructed by combining graphic matroids, co-graphic matroids, and a certain ten-element matroid $R_{10}$, see [26] or the book [25]. In the graphical case the last theorem says that the algebra remembers graph up to 2-isomorphism, see [32].

For the central case, we can extend Conjecture 8 for all matrices. For a matrix $A$, we say that a column is a bridge-column if after deleting it the rank decreases.

Conjecture 16. Let $A_{1} \in \mathbb{R}^{n_{1} \times m_{1}}$ and $A_{2} \in \mathbb{R}^{n_{2} \times m_{2}}$ be two matrices of ranks $n_{1}$ and $n_{2}$ respectively. Then the following are equivalent:

- $\mathcal{C}_{A_{1}}^{C}$ and $\mathcal{C}_{A_{2}}^{C}$ are isomorphic as non-graded algebras;

- $\mathcal{C}_{A_{1}}^{C}$ and $\mathcal{C}_{A_{2}}^{C}$ are isomorphic as graded algebras;

- $A_{1}^{\prime}$ and $A_{2}^{\prime}$ are $z$-equivalent, where $A_{i}^{\prime} \in \mathbb{R}^{\left(n_{i}-k_{i}\right) \times\left(n_{i}-k_{i}\right)}$ is the submatrix of $A_{i}$ resulting after deleting all $k_{i}$ bridge-columns and those $k_{i}$ rows such that $r k\left(A_{i}^{\prime}\right)=$ $n_{i}-k_{i}$.

\section{Proofs}

Let $B$ be a finite dimension of algebra over $\mathbb{R}$. We say that an element $r=\sum_{i=1}^{k} a_{2 i} a_{2 i+1}$ is reducible if $a_{i} \in B, i \in[2 k]$ are nilpotent elements.

For a nilpotent element $a \in B$, we define the length $\ell(a)$ as the maximal $\ell$ such that $a^{\ell} \neq 0$. 
Proof of Theorem 13. Clearly, we have $1 \Longleftarrow 2 \Longleftarrow 3$, so we will prove $1 \Longrightarrow 3$. Let $\mathcal{C}_{A}^{\mathcal{E} x}$ be our algebra. We will work with the square-free definition, i.e., $\mathcal{C}_{A}^{\mathcal{E} x}$ is a subalgebra of $\Phi_{m}$, where

$$
m=\max \left(\ell(a): a \in \mathcal{C}_{A}^{\mathcal{E} x}\right) .
$$

(Notice that at the moment we do not describe this embedding of $\mathcal{C}_{A}^{\mathcal{E} x}$ to $\Phi_{m}$, we know only its existence). We know which element is the unity, so we can choose a basis $x_{1}, \ldots, x_{n}$ of nilpotents of $\mathcal{C}_{A}^{\mathcal{E x}}$ with the following property:

$$
\ell(x+r) \geqslant \ell(x)
$$

for any reducible $r \in \mathcal{C}_{A}^{\mathcal{E} x}$ and $x \in \operatorname{span}\left\{x_{1}, \ldots, x_{n}\right\}$. Since we can define the algebra via some matrix $A$, then there is such a basis.

Any element has the representation

$$
x_{i}=\sum_{k=1}^{m} a_{i, k} \phi_{i}+r_{i},
$$

where $a_{i, k} \in \mathbb{R}$ and $r_{i}$ is reducible. Let $A^{\prime}=\left\{a_{i, k}:(i, k) \in[n] \times[m]\right\}$ be the corresponding matrix. Our goal is to reconstruct $A^{\prime}$ up to $z$-equivalece.

Consider the projective space $\mathbb{P}^{n-1}$ over $\mathbb{R}$. To finish the proof we should find the multiset

$$
\mathcal{A}:=\left\{\left(a_{1, k}, a_{2, k}, \ldots, a_{n, k}\right) \in \mathbb{P}^{n-1}, k \in[m]\right\} .
$$

Define the set $S$ of all non-zero $s \in \mathbb{R}$ such that there are $i \neq j \in[n]$ and a non-zero $t \in \mathbb{R}$ for which

$$
\ell\left(x_{i}-s x_{j}\right)<\ell\left(x_{i}-t x_{j}\right) .
$$

It is easy to see that $S$ is exactly the set

$$
\left\{\frac{a_{i, k}}{a_{j, k}}: i, j \in[n], k \in[m], \text { and } a_{i, k}, a_{j, k} \neq 0\right\} \text {. }
$$

Then $S$ is a finite set. Define the set $\mathcal{S}$ of possible elemnts of $\mathcal{A}$ as

$$
\mathcal{S}:=\left\{\left(s_{1}, s_{2}, \ldots, s_{n}\right) \in \mathbb{P}^{n-1}, s_{i} \in S \cup\{0\}\right\}
$$

Any element of $\mathcal{A}$ is an element of $\mathcal{S}$, so it is enough to find the multiplicity of any $s \in \mathcal{S}$.

Consider the following partial order on elements of $\mathbb{P}^{n-1}$ :

$$
\left(p_{1}, p_{2}, \ldots, p_{n}\right) \geqslant\left(p_{1}^{\prime}, p_{2}^{\prime}, \ldots, p_{n}^{\prime}\right)
$$

if there is $t \in \mathbb{R}$ such that

$$
\forall i \in[n], p_{i}^{\prime}=\left\{\begin{array}{l}
t p_{i} \\
0 .
\end{array}\right.
$$


Note that if, for any $s$, we know the common multiplication of all $s^{\prime} \geqslant s$ in $\mathcal{A}$ (the sum of multiplications), then we can calculate multiplicity of all elements.

Given $s \in \mathcal{S}$, then the summary multiplication of all $s^{\prime} \geqslant s$ is equal to

$$
\ell\left(\sum_{i \in I} b_{i} x_{i}\right)-\ell\left(\sum_{i \in I} c_{i} x_{i}\right)
$$

where

- $I \subseteq[n]$ is the support of $s$;

- $b_{i}, i \in I$ are generic;

- $c_{i}, i \in I$ are generic with the linear condition $\sum_{i \in I} c_{i} s_{i}=0$.

Let us check it:

$$
\begin{array}{r}
\ell\left(\sum_{i \in I} b_{i} x_{i}\right)=\ell\left(\sum_{i \in I} b_{i}\left(\sum_{k=1}^{m} a_{i, k} \phi_{k}+r_{k}\right)\right)=\ell\left(\sum_{i \in I} b_{i}\left(\sum_{k=1}^{m} a_{i, k} \phi_{k}\right)\right)= \\
=\ell\left(\sum_{k=1}^{m}\left(\sum_{i \in I} b_{i} a_{i, k}\right) \phi_{k}\right)=\#\left\{i \in k: \sum_{i \in I} b_{i} a_{i, k} \neq 0\right\},
\end{array}
$$

Similarly we have

$$
\ell\left(\sum_{i \in I} c_{i} x_{i}\right)=\#\left\{i \in k: \sum_{i \in I} c_{i} a_{i, k} \neq 0\right\} .
$$

Since $b_{i}, i \in I$ and $c_{i}, i \in I$ are generic with one condition $\sum_{i \in I} c_{i} s_{i}=0$, we have the following property: if $\sum_{i \in I} b_{i} a_{i, k} \neq 0$ then $\sum_{i \in I} c_{i} a_{i, k}=0$ if and only if $\left(a_{1, k}, \ldots, a_{n, k}\right) \geqslant s$.

Hence, we can compute the multiplicity of any $s$.

Proof of Theorem 15. We know $1 \Longleftrightarrow 2 \Longleftrightarrow 3 \Longrightarrow 4$ by Theorem 13, where we also reconstructed a matrix, so we know the matroid.

$3 \Longleftarrow 4$. Let $A_{1}$ and $A_{2}$ be two totally unimodular matrices which give the same regular matroid (we assume that the order of elements are the same).

Also if $M$ is a regular matroid, then all orientations of $M$ differ only by reorientations (see Corollary 7.9.4 [8]). Hence, we can multiply some columns of $A_{2}$ by -1 and get $A_{2}^{\prime}$ such that $A_{1}$ and $A_{2}^{\prime}$ have the same oriented matroid.

It is well-known that if we have a totally unimodular matrix $A_{i}$, then all minimal linear dependents of its columns have coefficients \pm 1 . We get that matrices $A_{1}$ and $A_{2}^{\prime}$ have linear dependents with the same coefficients and, hence, $A_{1} \sim_{z} A_{2}^{\prime} \sim_{z} A_{2}$. 


\section{References}

[1] A. A. Akopyan and A. A. Saakyan. A system of differential equations that is related to the polynomial class of translates of a box spline. Mat. Zametki, 44(6):705-724, 1999.

[2] F. Ardila and A. Postnikov. Combinatorics and geometry of power ideals. Transactions of the AMS, 362(8):4357-4384, 2010.

[3] F. Ardila. Enumerative and algebraic aspects of matroids and hyperplane arrangements. Ph.D. thesis, Massachusetts Institute of Technology, 2003.

[4] V. I. Arnold. Remarks on eigenvalues and eigenvectors of Hermitian matrices, Berry phase, adiabatic connections and quantum Hall effect. Selecta Mathematica, 1(1):119, 1995.

[5] A. Berget. Products of linear forms and Tutte polynomials. European J. Combin., 31:1924-1935, 2010.

[6] A. Berget. Internal zonotopal algebras and the monomial reflection groups $G(m, 1, n)$. J. of Combinatorial Theory, Ser. A, 159:1-25, 2018.

[7] O. Bernardi. Tutte polynomial, subgraphs, orientations and sandpile model: new connections via embeddings. Electronic J. Combinatorics, 15(1), \#R109, 2008.

[8] A. Björner, M. Las Vergnas, B. Sturmfels, N. White, and G. M. Ziegler. Oriented matroids. Vol. 46 of Encyclopedia of Mathematics and its Applications, 1999.

[9] W. Dahmen and A. Micchelli. On the local linear independence of translates of a box spline. Studia Mathematica, 82(3):243-263, 1985.

[10] C. De Boor and A. Ron. On polynomial ideals of finite codimension with applications to box spline theory. J. of Math. Analysis and Applications, 158(1):168-193, 1991.

[11] C. De Concini and C.Procesi. Topics in hyperplane arrangements polytopes and box-splines. Universitext, Springer, New York, 2011.

[12] N. Dyn and A. Ron. Local approximation by certain spaces of exponential polynomials, approximation order of exponential box splines, and related interpolation problems. Transactions of the AMS, 319(1):381-403, 1990.

[13] O. Holtz and A.Ron. Zonotopal algebra. Advances in Mathematics, 227:847-894, 2011.

[14] O. Holtz, A. Ron, and Z.Xu. Hierarchical zonotopal spaces. Transactions of the AMS, 364(2):745-766, 2012.

[15] B. Huang. Monomization of Power Ideals and Generalized Parking Functions. https : //math.mit.edu/research/highschool/primes/materials/2014/Huang.pdf

[16] A. N. Kirillov and G. Nenashev. On $Q$-deformations of Postnikov-Shapiro algebras. Séminaire Lotharingien de Combinatoire, 78B.55, FPSAC, 2017.

[17] A. N. Kirillov and G. Nenashev. Unimodular zonotopal algebra. in preparation 
[18] D. J. Kleitman and K. J. Winston. Forests and score vectors. Combinatorica, 1(1):4954, 1981.

[19] M. Lenz. Zonotopal algebra and forward exchange matroids. Advances in Mathematics, 294:819-852, 2016.

[20] M. Lenz. Lattice Points in Polytopes, Box Splines, and Todd Operators. International Mathematics Research Notices, 2015(14):5289-5310, 2016.

[21] M. Lenz. Hierarchical Zonotopal Power Ideals. European J. of Combinatorics, 33:1120-1141, 2012.

[22] L. Nan and A. Ron. External zonotopal algebra. J. of Algebra and its Applications, 13(02):1350097, 2014.

[23] G. Nenashev and B. Shapiro. "K-theoretic" analog of Postnikov-Shapiro algebra distinguishes graphs. J. of Combinatorial Theory, Ser. A, 148:316-332, 2017.

[24] G. Nenashev. Postnikov-Shapiro algebras, graphical matroids and their generalizations. arXiv:1509.08736, 2015.

[25] J. G. Oxley. Matroid theory. Oxford University Press, USA, 2006.

[26] P.D. Seymour. Decomposition of regular matroids. J. of Combinatorial Theory, Ser. B, 28(3):305-359, 1980.

[27] A. Postnikov and B. Shapiro. Trees, parking functions, syzygies, and deformations of monomial ideals. Transactions of the AMS, 356(8):3109-3142, 2004.

[28] A. Postnikov, B. Shapiro, and M. Shapiro. Algebras of curvature forms on homogeneous manifolds. "Differential Topology, Infinite-Dimensional Lie Algebras, and Applications: D. B. Fuchs 60th Anniversary Collection" AMS Transl., Ser. 2, 194:227235, 1999.

[29] B. Shapiro and M. Shapiro. On the ring generated by Chern 2-forms on $\mathrm{SL}_{n} / B$. Comptes Rendus de l'Academie des Sciences-Ser. I-Math., 326(1):75-80, 1998.

[30] J. J. Shan. A special case of Postnikov-Shapiro conjecture. arXiv:1307.5895, 2013.

[31] B. Sturmfels and Z.Xu. Sagbi bases of Cox-Nagata rings. J. of the EMS, 12(2):429459, 2010.

[32] H. Whitney. 2-isomorphic graphs. American J. of Mathematics, 55:245-254, 1993. 